\title{
MANY-ELECTRON EFFECTS IN ELECTRON-IMPACT IONIZATION OF MULTIPLY CHARGED IONS
}

\author{
R. A. Phaneuf \\ Oak Ridge National Laboratory* \\ Oak Ridge, TN 37831
}

\author{
Proceedings of Second International Workshop \\ on Cross Sections for Fusion and Other Applications \\ College Station, Texas \\ November 8-10, 1984 \\ To be published in \\ Nuclear Instruments and Methods in Physlcs Research \\ April 1985
}

\section{DISCLAIMER}

\begin{abstract}
This report was prepared as an account of work sponsored by an agency of the United States Government. Neither the United States Government nor any agency thereof, nor any of their employees, makes any warranty, express or implied, or assumes any legal liability or responsibility for the accuracy, completeness, or usefulness of any information, apparatus, product, or process disclosed, or represents that its use would not infringz privately owned rights. Reference herein to any specific commercial product, process, or service by trade name, trademark, manufacturer, or otherwise does not necessarily constitute or imply its endorsement, recommendation, or favoring by the United States Government or any agency thereof. The views and opinions of authors expressed herein do not necessarily state or reflect those of the United States Government or any agency thereof.
\end{abstract}

\footnotetext{
*Th1s research was supported by the office of Fusion Energy of the
} U.S. Department of Energy under contract No. DE-AC05-840R21400 with Martin Marietta Energy Systems, Inc. 


\title{
MANY-ELECTRON EFFECTS IN ELECTRON-IMF:ACT IONIZATION \\ OF MULTIPLY CHARGED IONS
}

$$
\text { R. A. Phaneuf }
$$

Oak Ridge National Laboratory

Oak Ridge, TN 37831

\begin{abstract}
Many-electron phenomena critically influence the ionization of multiply charged lons by electron 1mpact. In general, inner-shell electrons play an increasingly important role in the electron-impact Ionization process as the ionic or nuclear charge increase along an ionic sequence. A few specific examples are given to illustrate the signature and relative contributions of a number of indirect Ionization mechanisms to the total ionization cross sections.
\end{abstract}

\section{INTRODUCTION}

The stimulus of the fusion program and the availability of Improved Ion sources continue to produce a significant expansion of the base of accurate experimental data for electron-impact ionization of multiply charged ions. Systematization of experimental and theoretical studies along isoelectronic (same electronic structure), isonuclear (same species) or Isoionic (same charge) sequences have identified new Ionization mechanisms and produced an enhanced level of understanding of the fonization process. In many cases, the cross section for these so-called indirect Ionization channels dominate by more than an order of magnitude that for direct ejection of an outer-shell electron. These indirect mechanisms may be generally classified as many-electron effects, gince in each case, more 
than one Inftially-bound Ionic electron plays a role in the Ionization process via the autoionization or Auger process. There is also some experimental suggestion of correlated behavior of tonic electrons in multiple ionization in a single collision.

In this paper, a few examples will be given which illustrate the role of several different ionization mechanisms, and an attempt will be made to characterize some of the trends which are emerging from systematic studies along lonic sequences.

\section{IONIZATION MECHANISMS}

a. Direct Ionization

Direct Ionization refers to a transition from a bound state directly to the continuum, with no other excited bound or quasi-bound levels playing a role. The projectile electron directly ejects an electron from the lon, most commonly from the outer or valence shell:

$e+x^{+q}+e+x+q+n+n e$

This usually produces a single ionization event $(n=1)$, since the probability of a profectile electron causing the direct ejection of more than one ionic electron is relatively sma11. The direct ejection of an inner-shell electron leaves the lonized ion in an excited state. The process is usually classified as direct lonization if the "hole" state decays radiatively rather than by subsequent Auger emission.

Direct ionization cross sections may be estimated quite reliably by first-order quantum-mechanical perturbation treatments such as the distorted-wave Born approximation $[1,2]$. The Lotz semiempirical formula [3] is also widely used, and has been shown to predict direct Ionization 
cross sections to much better than a factor of two in most cases. A typlcal example relevant to fuston research is shown in Figure 1, where experimental cross sections [4] for electron-impact ionization of $\mathrm{Fe}^{2+}$ are compared to distorted-wave calculations [5] and to the Lotz prediction. The agreement in magnitude, and particularly in shape with the distortedwave calculation is favorable, except at the lowest energies, where the onset of the experimental Ionization cross section reflects the poyulation In the reacting beam of low-lying metastable levels within the $3 \mathrm{~d}^{6}$ grounds tate configuration.

b. Ionization-Autolonization

As noted above, the ejection of a more tightly bound inner-shell

electron leaves an excited ion which can subsequently autolonize, resulting in the ejection of one or more additional electrons, depending on the excitation energy of the "hole" state, and the branching ratio for radiative versus Auger decay (fluorescence yield). This indirect process is usually referred to as Ionization-autotonization, and is the dominant mechanism for net multiple Ionization in most cases:

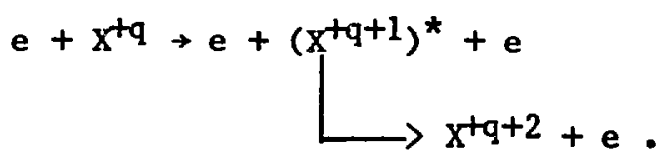

In this example, the excited state $\left(x^{+q+1}\right)$ decays by single autoionization ylelding net double ionization. For deeper izner-shell vacancies, successive Auger emissions, or simultaneous multiple Auger emissions may also be possible, resulting in net multiple lonization.

Figure 2, taken from a recent paper of PIndzola et al. [6], compares experimental cross sections $[6,7]$ for electron-impact double ionization in 
the isonuclear sequence $\mathrm{Ar}^{2+}, \mathrm{Ar}^{3+}$, and $\mathrm{Ar}^{4+}$. The comparison demonstrates the increasing dorinance of the $2 p$ iontzation-autolonization mechanism in the net double-ionization cross section relative to direct-double lonization as the Ionic charge Increases. This trend is expected to continue along the isonuclear sequence through $\mathrm{Ar}^{6+}$, with the double ionization cross section remaining roughly constant in magnitude, and equal to that for $2 p$ Ionization. The double-ionization threshold of $\mathrm{Ar}^{7+}$ increases to $566 \mathrm{eV}$, and ejection of a 2p electron can no longer produce double ionization via the Ionization-autoionization mechanism.

A number of possible lonization-autoionization channels in the multiple Ionization of $\mathrm{Xe}^{6+}$ are shown in Figure 3, where calculated Hartree-Fock energy thresholds [8] for the production of various innershell and multiple outer-shell vacancles are Indicated relative to the $3 d^{10} 4 s^{2} 4 p^{6} 4 d^{10} 5 s^{2}$ ground state of $X e^{6+}$. For example, ejection of a $4 d$ electron from $\mathrm{Xe}^{6+}$ can lead only to single ionization, whereas $4 \mathrm{p}$ ionization can lead to double ionization via the Ionization-autolonization mechanism. Figure 4 compares experimental cross sections [9] for single, double, and triple ionization of $\mathrm{Xe}^{6+}$ with Lotz predictions for the sum of cross sections for $5 s$ and $4 d$ lonization for $\sigma_{67}$, for $4 s$ and $4 p$ lonization for $\sigma_{68}$, and $3 d$ ionization for $\sigma_{69^{\circ}}$. The single lonization cross section, $\sigma_{67}$, is dominated by excitation-autoionization channels, and will be discussed in the following section. The experimental double-ionization cross section onset is consistent with $195 \mathrm{eV}$, which is the minimum energy required to remove two 5 s electrons, and significantly below the 4p ionization-autoionization threshold of $242 \mathrm{eV}$. Simllarly, the triple Ionization cross section, $\sigma_{69}$, shows an onset which is significantly below 
the 3d Ionization threshold of $764 \mathrm{eV}$, which is the lowest ionizationautolonization channel which can produce net triple ionization. Thus, for both $\sigma_{68}$ and $\sigma_{69}$, there is experimental evidence for the direct ejection of two or three outer-shell electrons. Cross sections for such a "direct multiple knockout" mechanism may be estimated using the semiclassical binary-encounter approximation [10], and are predicted to be negligible compared to the observed cross sections in both cases. The mechanisms which are responsible for these observations are not understood, but are suggestive of correlated or collective behaviot of the lonic electrons.

c. Excitation-Autoionization

Contributions to the Ionization of a multiply charged Ion produced by the excitation of an toulc Inner-shell electron to an autoionizing state were first Identified for Li-like lons by Crandall et al. [11]. This mechanism may be represented as follows:

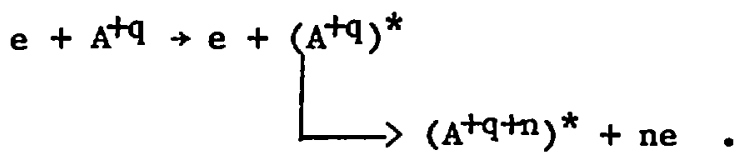

A number of examples of this mechanism have been documented, and it has been shown to dominate the single ionization cross sections for $\mathrm{Ti}^{3+}, \mathrm{Zr}^{3+}$, and $\mathrm{Hf}^{3+}$ by more than an order of magnitude [12]. Reasonable agreement has been obtained with experiment in many cases by simply adding theoretical cross sections for the appropriate excitation process to the direct lonization cross section, ignoring possible interference effects between these channe1s. Because the cross section for excitation of an ion by electron impact is finite and most often largest at its threshold energy, the excitation-autoionization process for a strong, well-isolated transition 
produces a distinctive and readily identiflable signature in the ionization cross section. In Figure 5, taken from a recent paper by Griffin et al. [13], experimental cross sections for five members of the Xe-1sonuclear sequence are compared to distorted-wave theoretical calculations for direct Ionization. Although the peak experimental cross sections decrease slowly with increasing ionic charge, they become increasingly larger than the direct-ionization theory as the lonic charge increases from 2 to 6 . This enhancement is attributed to inner-shell excitations to autoionizing levels of the types $X e^{+q}\left(4 d^{10} 5 s^{2} 5 p^{6-q}\right)+X e^{+q}\left(4 d^{9} 5 s^{2} 5 p^{6-q} n \ell\right)$ with $n \ell=4 f, 5 p$, 5d, and 5f. Hartree-Fock atomlc structure calculations for the $4 d^{9} 5 s^{2} 5 p^{6-q}$ ff configurations are highly term-dependent, and this was found to have a pronounced effect on the magnitude and energy dependences of the distorted-wave calculations of cross sections for these excitationautoionization contributions. Very reasonable agreement was realized between detailed theoretical calculations and experiment for these isonuclear lons, both with regard to the excitation energies and the magnitudes of the cross sections for the various excitations. The pronounced "hump-like" features in $\mathrm{Xe}^{2+}$ and $\mathrm{Xe}^{3+}$ occur because of the dominance of non-dipole-allowed excitations for these cases. The cross sections for such transitions are expected to fall off asymptotically as $1 / E$ for dipoleforbidden excitations, $a r_{i}$ as $1 / E^{3}$ for spin-forbidden excitations, whereas cross sections for both dipole-allowed excitations and for direct 1onization fall off much more slowly, as $\ell \mathrm{n} \mathrm{E/E} \mathrm{[14].} \mathrm{Thus} \mathrm{the} \mathrm{signature} \mathrm{of} \mathrm{an}$ excitation feature in the ionization cross section gives useful information about both the threshold energy and the nature of the excitation process which leads to autoionization. Coupled with a branching ratio for 
autolonization versus radiative decay, this signature can provide a quantitative basis for evaluating theoretical methods for electron-impact excitation cross section calculations. This can be particularly valuable, since direct excitation cross section measurements for lons, and particularly multiply charged lons, are technically very difficult, and few in number.

\section{d. Resonant-Recombination Double Autoionization}

In 1981, LaGattuta and Hahn [15] postulated an Ionization mechanism whereby the projectile electron resonantly recombines with the target ion to form a doubly-excited intermediate state, which subsequently decays by two-step Auger emission:

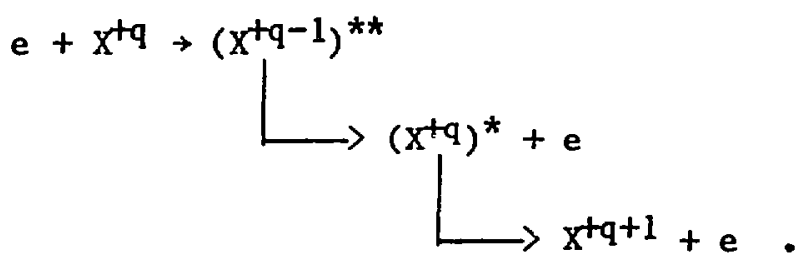

This seemingly exotic ionization mechanism was predicted to make a nonnegligible contribution to the total ionization cross section for $\mathrm{Fe}^{\mathrm{l} 5+}$. Henry and Msezane [16] have suggested a variation whereby the doublyexcited intermediate state decays by simultaneous double Auger emission, which they have labeled "auto-double Ionization."

The resonant process here is analogous to dielectronic recombination, except that it is associated with the excitation of an inner-shell electron. Assume that an electron approaches an 1on with just less than the energy required to excite a transition in the ion. Kinetic-energy gain due to acceleration by the Coulomb field of the fon may allow the electron to excite the ion, but the electron is now trapped by the Coulomb field, and Is captured Into a doubly-exc1ted Rydberg level. The doubly excited ton 
can elther radiate away some of the excess energy, stabilizing the recombination, or can autoionize. If the excitation involves an inner-shell electron, sequential or simultaneous double autolonization may be energetically possible. The signature for this process is expected to be the same as that for dielectronic recombination, $1 . e .$, the cross section consists of a Rydberg series of sharp resonances just below and converging to the threshold energy of the relevant excitation. The expected effect on an experimental electron-impact ionization cross section measurement with finite electron energy resolution is a smearing-out of the sharp onset of an excitation feature to energies below the expected excitation threshold. In Figure 6, taken from ref. [13], theoretical distorted-wave calculations for both direct lonization and excitation-autoionization are summed and compared to the experimental data. Experiment and theory are in nearly perfect agreement at energies above the cross-section maximum at $85 \mathrm{eV}$, but the experimental ionization cross section shows significant enhancement over direct Ionization at energies below the range of predicted excltation thresholds. Although no specific calculations have yet been performed, similar features are present in other members of the Xe isonuclear sequence, and are suggestive of the resonant mechanism.

\section{SUMMARY}

Many-electron phenomena play an Important role in the electron-impact ionization of multiply charged 1ons. Through systematic experimental and theoretical investigations along ionic sequences, a number of indirect Ionization mechanisms have been identified and accurately quantified in many cases. In general, as the ionic charge increases along an isonuclear 
sequence, these indirect Ionization channels and the role of inner electronic shells become progressively more important. Similarly, multiple Ionization is dominated by inner-shell ionization-autoionization and becomes relatively more important with increasing lonic charge. There is some indication that correlated or collective electron behavior may in some cases play a role in multiple ionization by electron impact. Experimental evidence also suggests that more exotic indirect Ionization mechanisms such as resonant-recombination multiple autoionization may also make a significant contribution to the total ionization cross section in some cases. The Increasing level of systematic experimental and theoretical activity on this subject promises to resolve some of these puzzles, and uncover new ones in the process.

\section{ACKNOWLEDGMENTS}

The author is indebted to D. C. Gregory, A. M. Howald, and D. H. Crandall, who are or have been recent members of the ORNL electron-ion experimental team. A number of these measurements were performed in continuing collaboration with G. H. Dunn and members of his JILA research group (D. W. Mueller and T. J. Morgan).

The ORNL theoretical atomic physics for fusion group of C. Bottcher, D. C. Griffin, and M. S. Pindzola continue to provide the theoretical background and much of the inspiration for this work. This research was supported by the Office of Fusion Energy of the U.S. Department of Energy under contract No. DE-ACO5-840R21400 with Martin Marietta Energy Systems, Inc. 
REFERENCES

1. S. M. Younger, Phys. Rev. A 22, 111 (1980).

2. M. S. Pindzola, D. C. Griffin, and C. Bottcher, J. Phys. B 16, 2355 (1983).

3. W. Lotz, Z. Physik 206, 205 (1967); ibid 220, 466 (1969).

4. D. W. Mueller, T. J. Morgan, G. H. Dunn, D. C. Gregory, and D. H. Cranda11, "Absolute cross section measurements for electron-impact Ionization of twice-charged ions: $\mathrm{Ti}^{2+}, \mathrm{Fe}^{2+}, \mathrm{Ar}^{2+}, \mathrm{Cl}^{2+}$, and $\mathrm{F}^{2+}$, Phys. Rev. A (submitted 1984).

5. M. S. Pindzola, D. C. Griffin, C. Bottcher, D. C. Gregory, A. M. Howald, R. A. Phaneuf, D. H. Crandall, G. H. Dunn, D. W. Mueller, and T. J. Morgan, Oak Ridge National Laboratory Report ORNL/TM-9436 (January 1985).

6. M. S. Pindzola, D. C. Griffin, C. Bottcher, D. H. Crandall, R. A. Phaneuf, and D. C. Gregory, Phys. Rev. A 29, 1749 (1984).

7. A. Müller and R. Frod1, Phys. Rev. Lett. 44, 29 (1980).

8. M. S. Pindzola, private communication (1983).

9. A. M. Howald, D. C. Gregory, D. H. Crandall, and R. A. Phaneuf, unpublished data, ORNL (1984).

10. M. Gryzinski, Phys. Rev. 138, A336 (1965).

11. D. H. Crandall, R. A. Phaneuf, B. E. Hasselquist, and D. C. Gregory, J. Phys. B 12, L249 (1979).

12. R. A. Falk, G. H. Dunn, D. C. Gregory, and D. H. Crandal1, Phys. Rev. A 27, 762 (1983).

13. D. C. Griffin, C. Bottcher, M. S. Pindzola, S. M. Younger, D. C. Gregory, and D. H. Cranda11, Phys. Rev. A 29, 1729 (1984). 
14. W. D. Robb, "Theoretical Studies of Electron Impact. Ionization," pp. 245-265 in Atomic and Molecular Processes in Controlled Thermonuclear Fusion, eds., M.R.C. McDowell and A. M. Ferendeci, Plenum, New York, 1980.

15. K. J. LaGattuta and Y. Hahn, Phys. Rev. A 24, 2273 (1981).

16. R.J.W. Henry and A. Z. Msezane, Phys. Rev. A 26, 2545 (1982). 


\section{FIGURE CAPTIONS}

Figure 1. Experimental and theoretical cross sections versus electron energy for electron-impact ionization of $\mathrm{Fe}^{2+}$. The dashed curve represents the Lotz semiempirical formula [3] and the solid curve the Coulombdistorted-wave calculation of Pindzola et al. [5] for direct ionization. The experimental data are those of Mueller et al. [4].

Figure 2. Comparison of electron-impact double-ionization cross sections in the Ar isonuclear sequence, from ref. 6. The open circles and triangles are experimental data of Mïller et al. [7] for $\mathrm{Ar}^{2+}$ and $\mathrm{Ar}^{3+}$, respectively; and solid circles are measurements of Crandall et al. [6] for Ar ${ }^{4+}$. Solid curve is a distorted-wave calculation of Pindzola et al. [6] for ionization of a $2 \mathrm{p}$ electron in $\mathrm{Ar}^{4+}$.

Figure 3. Hartree-Fock theoretical energy thresholds from Pindzola [8] for Ionization of various subshells in the Xe isonuclear sequence. The energy scale is relative to the $3 \mathrm{~d}^{10} 4 \mathrm{~s}^{2} 4 \mathrm{p}^{6} 4 \mathrm{~d}^{10} 5 \mathrm{~s}^{2}$ ground state of $\mathrm{Xe} \mathrm{e}^{6+}$. The tops of the cross-hatched bars designate the ionization potentials of each stage, and downward arrows designate possible autoionizition pathways.

Figure 4. Experimental cross sections versus electron energy [9] for electron-impact single $\left(\sigma_{67}\right)$, double $\left(\sigma_{68}\right)$, and tripie $\left(\sigma_{69}\right)$ ionization of $\mathrm{Xe}^{6+}$. The vertical arrows designate the minimum threshold energies for each process. The dashed curves are the sum of Lotz semlempirical cross sections for 5 s and $4 d$ ionization which contribute to $\sigma_{67}$, for $4 s$ and $4 p$ ionization which contribute to $\sigma_{68^{\circ}}$, and for $3 d$ ionization in the case of $\sigma_{69^{\circ}}$ 
Figure 5. Comparison of experidental cross sections and distortedwave direct-ionization calculations for electron-impact single lonization in the Xe-isonuclear sequence, from Griffin et al (ref. 13).

Figure 6. Comparison of experimental and theorețlcal cross sections for electron-impact ionization of $\mathrm{Xe}^{3+}$ near threshold. Long-dashed curve is the distorted-wave direct ionization calculation. Solid curve represents distorted-wave cross sections for excitation autoionization added to the direct cross section. The short-dashed curve is a convolution of the solid curve with a $2 \mathrm{eV}$ FWIM Gaussian to model the finite experimevical energy spread. Arrows designate ranges of threshold energies for the excitations to autoionizing levels in the configurations indicated. 


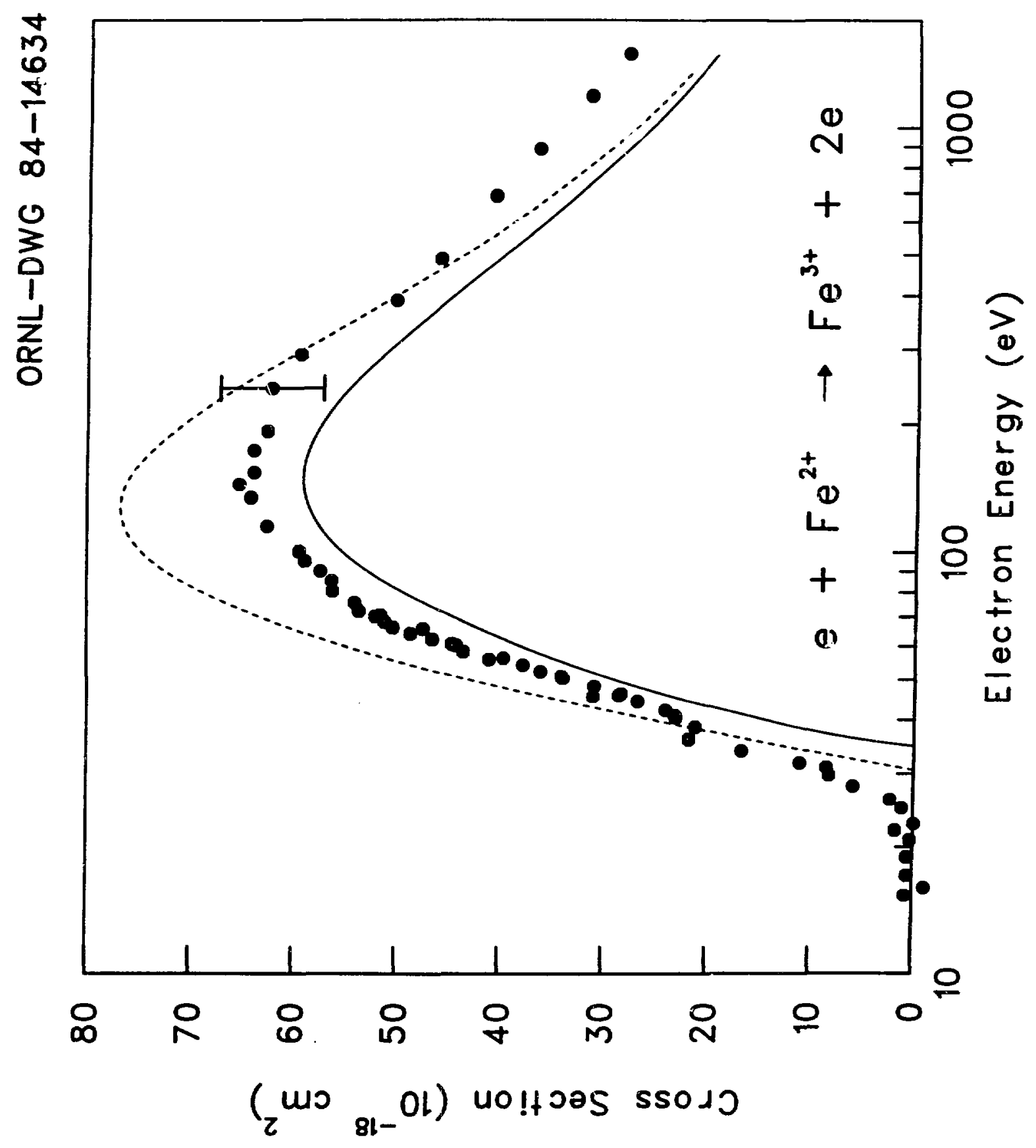




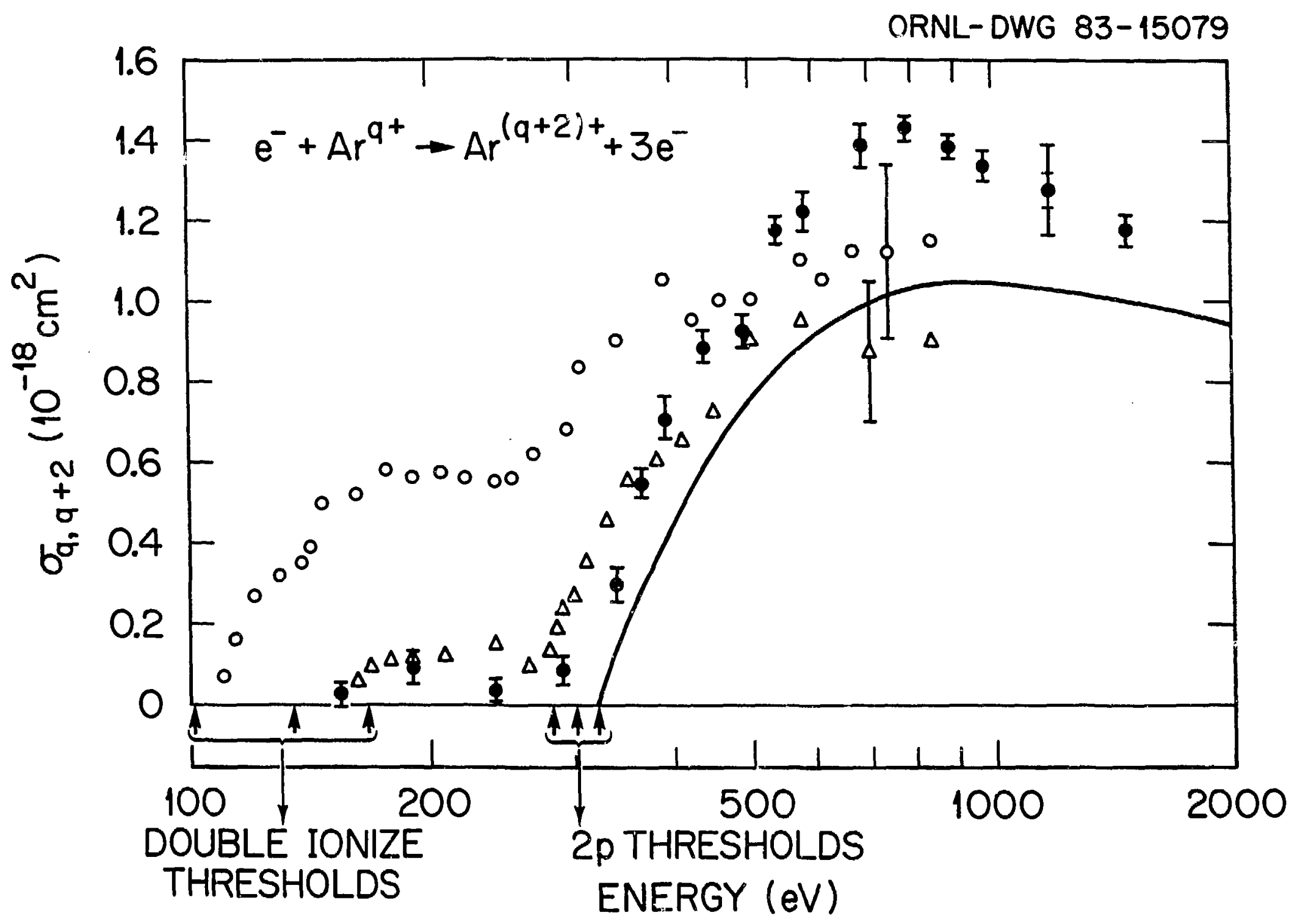


Fig. 3

ORNL- OWG 84-10641

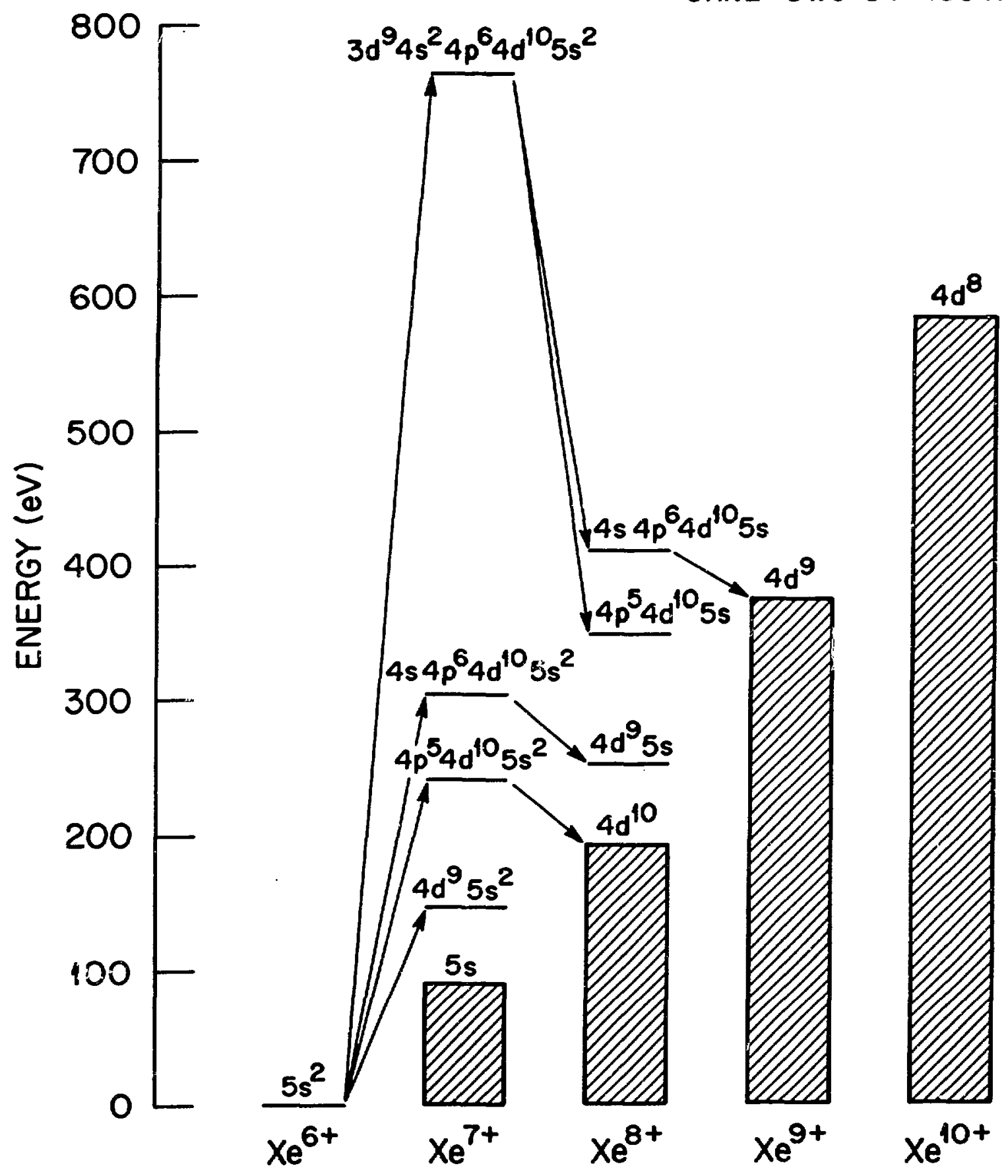


Fig. 4

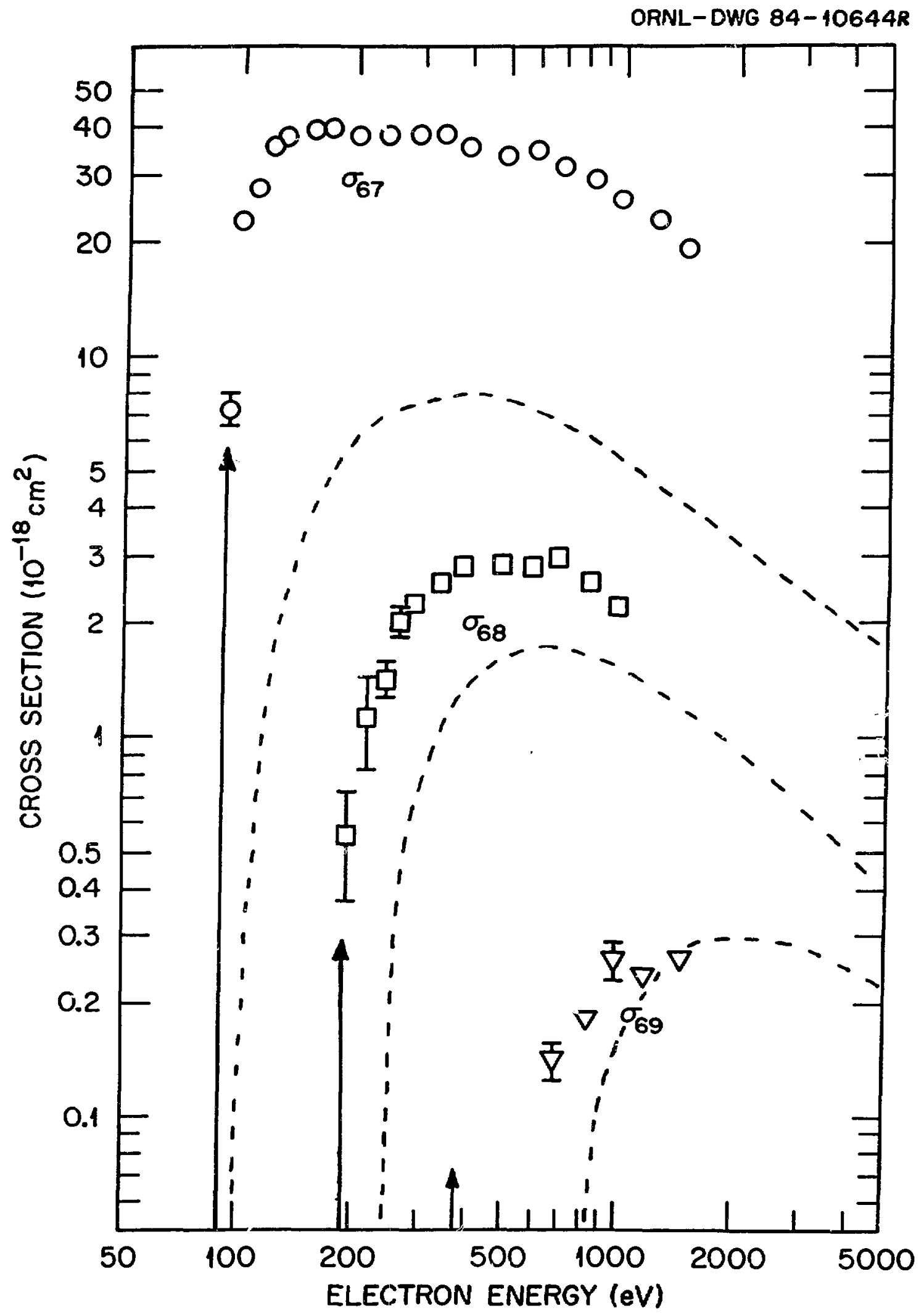


Fig. 5
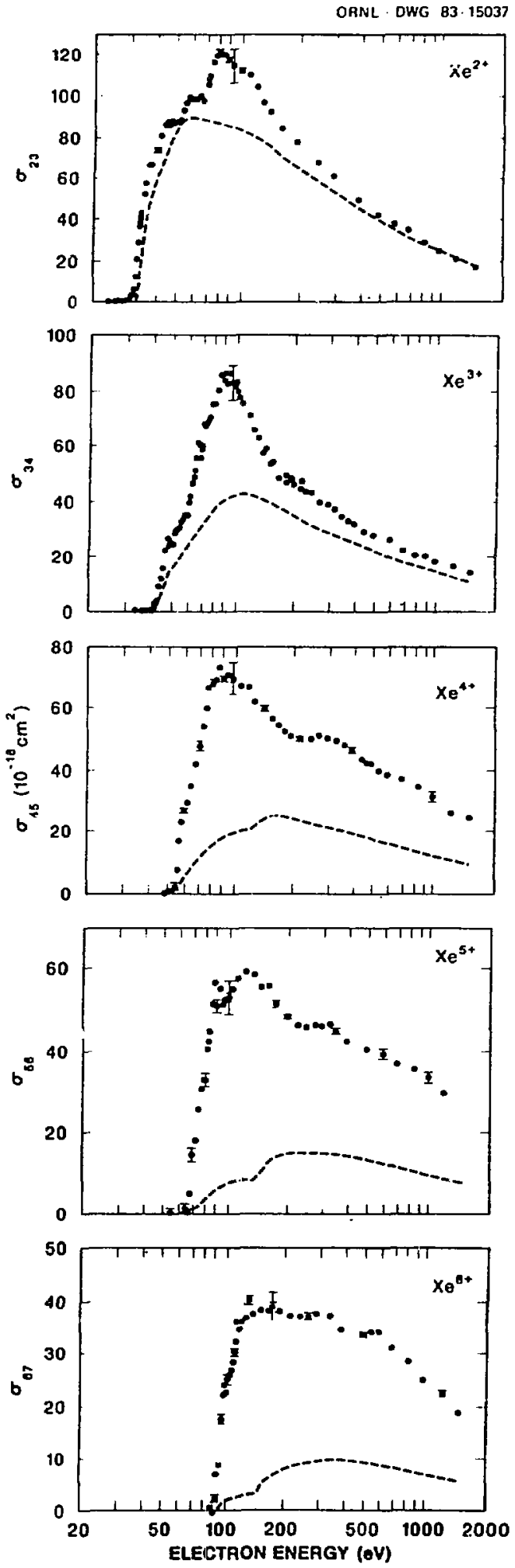
$\omega$
$\dot{\omega}$

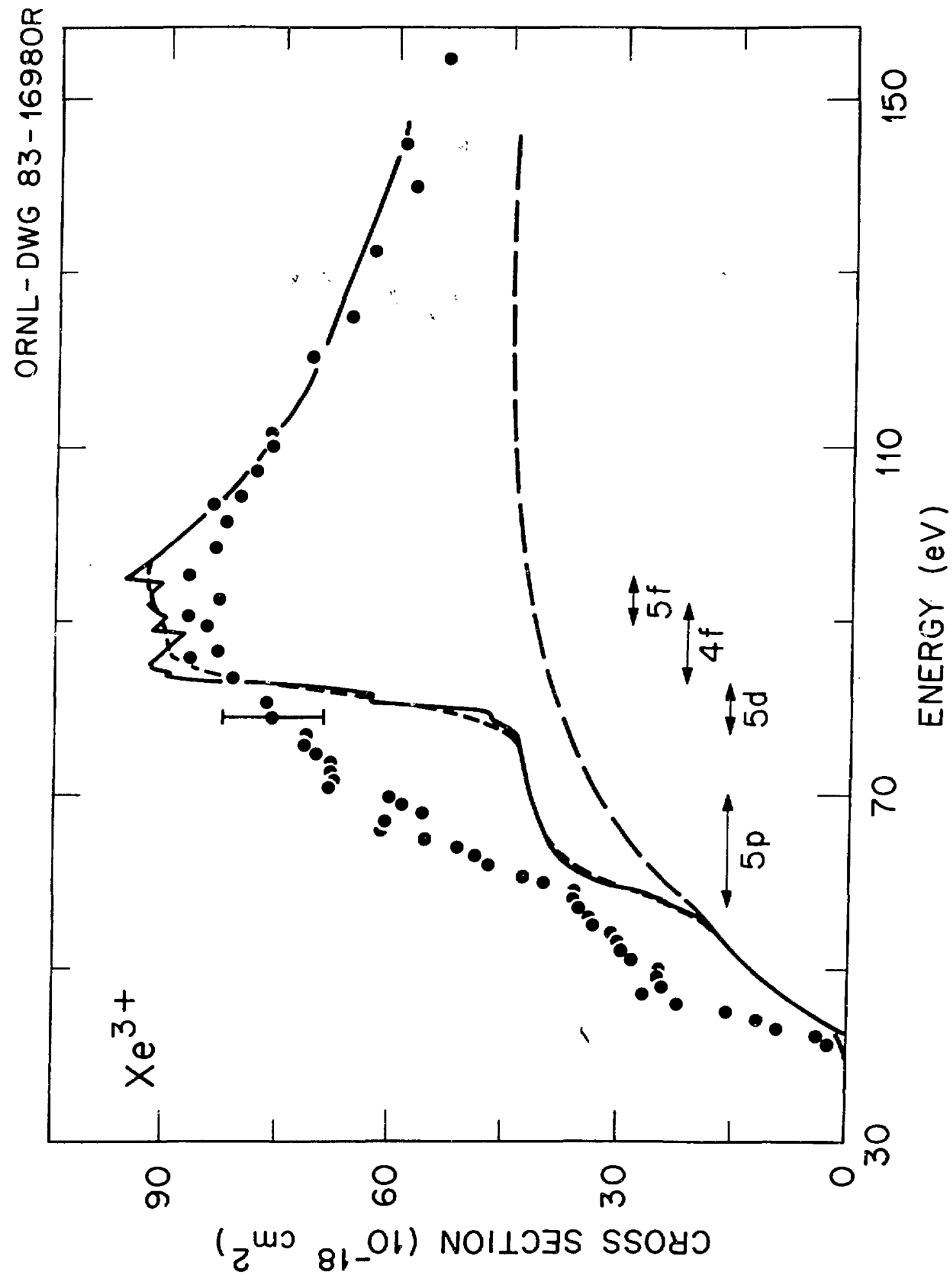

